\title{
ANÁLISE QUALI-QUANTITATIVA DAS POPULAÇÕES ALGÁCEAS DE UM TRECHO RECIFAL NA PRAIA DE BOA VIAGEM, PE
}

Fernanda Alves Ribeirol, Antônio Travassos Júnior ${ }^{l}$, Lísia Mônica Gestinari' ${ }^{l}$, Juliana Torres ${ }^{l}$, Karina Kelly dos Anjos Lima', Maria das Dores dos Santos ${ }^{l}$, Giulliari Allan S. T. Lira', Khey Albert A. Fontes ${ }^{1}$, Sonia Maria Barreto Pereira ${ }^{l^{*}} \&$ Yocie Yoneshigue Valentin ${ }^{2}$

1 Universidade Federal Rural de Pernambuco (UFRPE), Departamento de Biologia, Programa de Pós-Graduação em Botânica (PPGB). Av. Dom Manuel de Medeiros, s/n, Dois Irmãos, 52171-900, Recife, PE, Brasil.

2 Universidade Federal do Rio de Janeiro, Centro de Ciências da Saúde, Instituto de Biologia, Departamento de Botânica, Av. Brigadeiro Trompowsky, $\mathrm{s} / \mathrm{n}^{\circ}$, Ilha do Fundão, 21941-900, Rio de Janeiro, RJ, Brasil.

*E-mail: soniabp@terra.com.br

\section{RESUMO}

Este trabalho analisa a estrutura das populações de macroalgas da praia de Boa Viagem ( $08^{\circ} 05^{\prime 2} 26^{\prime \prime}$; $34^{\circ} 52^{\prime} 55^{\prime}$ 'W e $08^{\circ} 08^{\prime} 52^{\prime \prime} \mathrm{S}$; 345'ㄱ'W), localizada no município de Recife (PE). A metodologia utilizada foi a dos "Relevés" fitossociológicos. No platô recifal da região entre marés foram demarcadas duas áreas denominadas de Pontos. O Ponto 1 localizado em uma área exposta e o Ponto 2 localizado em uma área abrigada. Foram realizadas coletas durante as estações chuvosa (abril 2002) e seca (novembro de 2003). Os seguintes parâmetros foram observados: Número de Táxons, Recobrimento de cada espécie (Ri), Recobrimento total (Rt), Diversidade (H'), Equitabilidade (J), Reprodutividade da espécie (Gi) e Densidade de reprodução calculada para cada "relevé" (dG). Foram identificadas 49 espécies, sendo 33 Rhodophyta, cinco Ochrophyta e 11 Chlorophyta. O recobrimento total variou entre 187,55\% (Ponto 1, período seco) a 345,35\% (Ponto 2, período seco). A diversidade apresentou valores entre 2,22 a 2,62 nos dois períodos sazonais e a equitabilidade variou entre 0,29 e 0,75. Esses resultados demonstram que as populações de macroalgas de Boa Viagem apresentam uma diversidade média. Os indivíduos encontram-se bem distribuídos entre as espécies, exceto no Ponto 2 (Lado Abrigado) no período chuvoso.

Palavras-chave: Algas bentônicas, recife, estrutura de populações, Pernambuco, Brasil.

\section{ABSTRACT}

QUALI-QUANTITATIVE ANALYSIS OF ALGAE POPULATIONS IN THE REEF COAST OF BOA VIAGEM, PERNAMBUCO/BRAZIL. This study analyzed the structure of seaweed populations

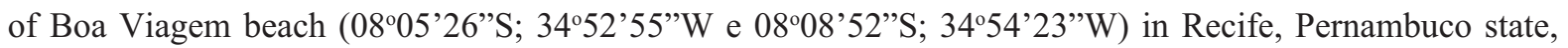
Brazil, through the phytosociologic relevé method. Two locations were selected from the reef plateau surface on the intertidal zone: location 1 was placed on the exposed side of the reef plateau and location 2 was placed on the sheltered side of the plateau. Samples were collected in a rainy period (April 2002) and in a dry period (November 2003). The following parameters were determined: number of taxa, coverage area of each species (Ri), total coverage area (Rt), diversity ( $\left.\mathrm{H}^{\prime}\right)$, evenness (J), species reproductivity (Gi) and density of reproduction for each "relevé" (dG). Forty-nine species were identified, being 33 Rhodophyta, five Ochrophyta and 11 Chlorophyta. The lowest total coverage area observed was $87.55 \%$ on location 1 during the dry season and the greatest was $345.35 \%$ on location 2 during the dry season. Diversity values varied 2.22-2.62 during the two periods and evenness varied 0.29-0.75. The results indicate the seaweed populations of Boa Viagem present intermediary diversity. The number of individuals among the different species was fairly balanced, except for the sheltered side of location 2 in the rainy season.

Keywords: Benthic algae, reef, population structure, Pernambuco, Brazil.

\section{INTRODUÇÃO}

O litoral de Pernambuco está situado na região tropical (Horta et al. 2001) correspondente a zona Oriental proposta por Oliveira Filho (1977). A referida região se caracteriza pela ocorrência de uma flora ficológica relativamente rica se desenvolvendo, sobretudo, nas formações recifais. Na costa do estado são encontradas extensas formações recifais (recifes de franja) constituídas por embasamento arenítico e

Oecol. Bras., 12 (2): 222-228, 2008 
arenítico-ferruginoso, recobertas por algas calcárias incrustantes e corais, constituindo-se, assim, em uma das principais áreas de diversidade de espécies do litoral nordestino (Pereira 2002, Pereira et al. 2002).

Apesar das características citadas, poucos são os trabalhos desenvolvidos na costa de Pernambuco que tratam dos aspectos fitossociológicos de populações algáceas destacando-se os de Silva et al. (1987), Ferreira et al. (1988), Muñoz \& Pereira (1998), Mansilla \& Pereira (2001), Pereira et al. (no prelo).

A praia de Boa Viagem é muito influenciada pela ação antrópica, principalmente, aquelas representadas por pisoteio no recife e uso de embarcações. Apesar de estar localizada em uma área urbana, apresenta uma alta variedade de espécies de algas bentônicas (Pereira et al. 2002), sendo necessário, portanto, estudos mais detalhados sobre a estrutura dessas populações.

Este estudo tem como objetivo caracterizar qualiquantitativamente as populações das algas pluricelulares de um trecho de recife da praia de Boa Viagem, descrevendo suas características fitossociológicas.

\section{MATERIAL E MÉTODOS}

\section{ÁREA DE ESTUDO}

A praia de Boa Viagem está localizada no município de Recife (0805'26"S, 3452'55"W e $\left.08^{\circ} 08^{\prime} 52^{\prime \prime} \mathrm{S}, 34^{\circ} 54^{\prime} 23^{\prime} \mathrm{W}\right)$, em região de clima Tropical Atlântico, com temperatura média de $26^{\circ} \mathrm{C}$. A precipitação anual média é de $2.000 \mathrm{~mm}$ e a distribuição das chuvas define dois períodos anuais: um seco, entre os meses de setembro a fevereiro, e um chuvoso entre março a agosto (Kempf 1967/69).

No local ocorrem cordões recifais, com aproximadamente $6 \mathrm{~km}$ de extensão, funcionando como um sistema natural de proteção direto à linha da costa, além de servirem como substrato para o desenvolvimento das algas, bem como de uma fauna associada bastante diversificada (Guerra \& Manso 2004). Nessas formações, em função dos níveis das marés, são encontrados andares (regiões) denominadas de mesolitoral e infralitoral. $\mathrm{O}$ mesolitoral está situado na parte anterior dos recifes no sentido leste, onde se encontra o compartimento do platô. O infralitoral, sempre imerso, está situado na parte posterior ao mesolitoral, iniciando a partir da zona de arrebentação (Oliveira-Carvalho et al. 2003).

\section{ETAPA DE CAMPO}

O trecho recifal para o desenvolvimento deste trabalho (0809'S e 345' $\mathrm{W}$ ) foi selecionado por apresentar uma largura maior no compartimento do platô (em torno de $30 \mathrm{~m}$ ) e porque as populações algáceas são homogêneas, proporcionando condições favoráveis para a realização das amostragens.

O método empregado no presente estudo foi o do "relevé", considerado um dos métodos mais completos para estudos quantitativos de vegetação algácea. Isso se deve a sua rigorosidade nas amostragens e o uso de parâmetros fitossociológicos analíticos e sintéticos. Segundo Yoneshigue \& Valentin (1988), um "relevé" consiste na coleta completa (método destrutivo) de toda a vegetação contida em um quadrado determinado, por meio de uma raspagem integral do substrato, incluindo as algas crostosas, o que destaca a importância de todos os estratos.

No platô recifal de Boa Viagem foram demarcadas duas áreas denominadas de Ponto 1 e Ponto 2. O Ponto 1 está localizado numa área considerada "exposta" (Lado Exposto) estando as populações algáceas sob constante influência da arrebentação das ondas. O Ponto 2 está localizado em uma área abrigada (Lado Abrigado) estando as algas protegidas do batimento direto das ondas. Em seguida, aferiu-se o grau de inclinação do substrato em relação à exposição ao sol. O Ponto 1 apresenta exposição oeste e $8^{\circ}$ de inclinação e o Ponto 2 com exposição sudeste e $20^{\circ}$ de inclinação do substrato. Foram realizadas duas coletas no Platô: uma no mês de abril de 2002 (estação chuvosa) e outra em novembro de 2003 (estação seca), durante as marés baixas.

Para a realização do "relevé" foi empregado um quadrado de $20 \times 20 \mathrm{~cm}$. Esta área, geralmente, é considerada superior a área mínima para fitobentos de águas temperadas ou quentes (Yoneshigue \& Valentin 1988).

As amostras coletadas foram acondicionadas em sacos de polietileno, devidamente etiquetados. Ainda no campo o material foi fixado em solução de formol (4\%) preparada com água do mar sendo, então, transportado para o Laboratório de Ficologia (LABOFIC) da Universidade Federal Rural de Pernambuco para posterior triagem e identificação. 


\section{ETAPA DE LABORATÓRIO}

No laboratório todo o material foi minuciosamente triado com uso de microscópio e estereoscópio ópticos. Foram excluídas as microalgas e a fauna acompanhante.

Para cada amostra foi elaborada uma lista de espécies e foram calculados os seguintes parâmetros analíticos, de acordo com Yoneshigue \& Valentin (1988):

$\mathrm{T}$ (número de táxons): número de espécies encontradas.

$\mathrm{Ri}$ (recobrimento da espécie): é a porcentagem de superfície do quadrado ocupada em projeção vertical pela espécie. Os indivíduos de cada espécie, após triados, foram dispostos em uma grade com a mesma área do quadrado $\left(400 \mathrm{~cm}^{2}\right)$ e dividida previamente em 100 sub-quadrados iguais. Assim, o Ri representa o número de sub-quadrados recobertos pela planta. Para espécies cujo recobrimento não pôde ser medido, sua presença foi registrada com o sinal +.

Rt (recobrimento total): é a soma de todos os Ri de todas as espécies.

H' (índice de diversidade específica);

$\mathrm{J}$ (Eqüitabilidade);

Gi (reprodutividade da espécie): para cada espécie encontrada foram montadas 10 lâminas com material escolhido ao acaso, no intuito de observar a presença ou ausência de órgãos reprodutores. Por definição, a reprodutividade de cada espécie pode ter os seguintes valores: 0 - sem órgãos reprodutores, 1 - órgãos reprodutores muito raros, 3 - órgãos reprodutores raros, 6 - órgãos reprodutores comuns, 10 - órgãos reprodutores muito comuns.

dG (densidade de reprodução calculada para cada "relevé") - $\sum^{\mathrm{T}}$ (Ri.Gi)/ $\mathrm{R}^{\mathrm{T}}$; este parâmetro permite quantificar o nível de reprodução da população.

Para identificar possíveis associações entre as espécies, foram feitos dendrogramas, com a utilização do programa PRIMER.

\section{RESULTADOS E DISCUSSÃO}

A partir da análise do quadrado de $20 \times 20 \mathrm{~cm}$ foram identificadas um total de 49 espécies, das quais 33 pertencem ao Phylum Rhodophyta (67,35\%), cinco ao Phylum Ochrophyta (10,2\%) e 11 ao Phylum Chlorophyta $(22,45 \%)$ (Tabela I).
De acordo com Pereira et al. (2002) as seguintes espécies são novas ocorrências para a costa de Pernambuco: Hypnea cenomyce, H. cornuta, Hecatonema floridanum e H. terminalis.

Silva et al. (1987), Mansilla \& Pereira (2001) e Pereira et al. (no prelo) estudando as populações de macroalgas de outros trechos de recifes pernambucanos encontraram um padrão florístico semelhante ao da praia de Boa Viagem, com o predomínio de Rhodophyta, seguido por Chlorophyta e Ochrophyta.

A presença dos gêneros Halimeda, Bryopsis e Caulerpa (entre as Chlorophyta) Dictyopteris e Padina (entre as Ochrophyta) e Gracilaria, Ceramium e Hypnea (entre as Rhodophyta), indicam o caráter tropical da flora ficológica da praia de Boa Viagem (Pereira et al. 2002, Bandeira-Pedrosa et al. 2004, Villaça et al. 2006).

Do total de espécies identificadas 21 foram exclusivas do Ponto 1 (Lado Exposto), duas exclusivas do Ponto 2 (Lado Abrigado) e 27 espécies ocorreram em ambos os pontos (Tabela I). Dentre as espécies exclusivas do Ponto 1 podem ser destacadas Plocamium brasiliense, Polysiphonia subtilissima, Sahlingia subintegra, Stylonema alsidii, Hecatonema floridanum, Hecatonema terminalis, Bryopsis sp. e Rhizoclonium riparium. Essas algas alcançaram seu maior desenvolvimento no período seco, em regiões parcialmente expostas nas áreas planas dos recifes. Isso ocorreu devido aos seus curto ciclo de vida e alto índice de reprodução (Costa Jr. et al. 2002).

Com relação à variação temporal, no período chuvoso, embora o Ponto 2 tenha apresentado um menor número de espécies, o recobrimento total foi maior que no Ponto 1, indicando a presença de indivíduos mais desenvolvidos. Entretanto, o Ponto 1 apresentou maiores valores para número de espécies e para os índices de diversidade e eqüitabilidade, além de uma maior densidade reprodutiva (Tabela II). Com relação ao período seco, o Ponto 1 apresentou, novamente, um maior número de espécies (37) enquanto no Ponto 2 foram identificados 18 táxons. Apesar de apresentar menos espécies o Ponto 2 apresentou uma maior cobertura (345,35\%). Em ambos os lados ocorreu um aumento da cobertura no período chuvoso. Silva et al. (1987) analisando a estrutura de um banco de Gracilaria sp., em Itamaracá/PE, encontraram um resultado semelhante, com uma maior biomassa durante 
Tabela I. Recobrimento (Ri) e reprodutividade (Gi) de cada espécie nos Pontos 1 e 2 do platô recifal da praia de Boa Viagem, Recife (PE) durante o período chuvoso (abril-2002) e seco (novembro-2003). O sinal + indica a presença da espécie. O sinal - indica a ausência da espécie.

Table I. Percentage coverage area (Ri) and reproductivity (Gi) of each species in location 1 and location 2 of the reef plateau of Boa Viagem beach in Recife, Pernambuco state, Brazil, during the rainy period (April 2002) and dry period (November 2003). A "+" indicates species was present, and a "- " indicates it was not found.

\begin{tabular}{|c|c|c|c|c|c|c|c|c|}
\hline & \multicolumn{4}{|c|}{ Ponto 1 (Lado Exposto) } & \multicolumn{4}{|c|}{ Ponto 2 (Lado Abrigado) } \\
\hline & \multicolumn{2}{|c|}{ Período chuvoso } & \multicolumn{2}{|c|}{ Período seco } & \multicolumn{2}{|c|}{ Período chuvoso } & \multicolumn{2}{|c|}{ Período seco } \\
\hline & $\operatorname{Ri}(\%)$ & $\mathrm{Gi}$ & $\operatorname{Ri}(\%)$ & Gi & $\operatorname{Ri}(\%)$ & $\mathrm{Gi}$ & $\operatorname{Ri}(\%)$ & Gi \\
\hline Acrochaetium $\mathrm{sp}$. & + & 0 & + & - & + & - & - & - \\
\hline $\begin{array}{l}\text { Aglaothamnion felipponei (M. Howe) N. } \\
\text { Aponte, D. L. Ballant. \& J. N. Norris. }\end{array}$ & - & - & + & - & - & - & 0,01 & 0 \\
\hline Amphiroa fragillissima (L.) J. V. Lamour. & 0,10 & 0 & - & - & - & - & - & - \\
\hline Bryocladia sp. & 0,04 & 0 & - & - & - & - & - & - \\
\hline Bryothamnion seaforthii (Turner) Kütz. & - & - & + & 0 & - & - & - & - \\
\hline $\begin{array}{l}\text { Centroceras clavulatum (C. Agardh in Kunth) } \\
\text { Mont. in Durieu de Maisonneuve }\end{array}$ & 0,09 & 0 & 0,74 & 0 & + & 0 & + & 0 \\
\hline Ceramium flaccidum (Kütz.) Ardiss. (epífita) & 6 & 6 & + & 5 & 3 & 10 & 0,6 & 2 \\
\hline Ceramium sp. (epífita) & - & - & 12,5 & 0 & + & 0 & - & - \\
\hline Chondracanthus acicularis (Roth) Fradericq & 0,25 & 0 & 9 & 0 & 4 & 0 & 6 & 0 \\
\hline $\begin{array}{l}\text { Chondrophycus papillosus (C. Agardh) } \\
\text { Garbary \& J. T. Harper }\end{array}$ & 12 & 6 & 10,33 & 1 & 28 & 0 & - & - \\
\hline Corallinaceae incrustante & 100 & 0 & 0,875 & 0 & 100 & 0 & 100 & 0 \\
\hline Erythrotrichia carnea (Dillwyn) J. Agardh & + & 0 & 0,01 & 0 & + & 0 & - & - \\
\hline $\begin{array}{l}\text { Gelidiella acerosa (Forssk.) Feldmann \& } \\
\text { Hamel }\end{array}$ & 70 & 6 & 45,33 & 1 & 70 & 0 & 73 & 0 \\
\hline $\begin{array}{l}\text { Gelidiopsis planicaulis (W. R. Taylor) W. R. } \\
\text { Taylor }\end{array}$ & 3 & 0 & - & - & - & - & 10,5 & 0 \\
\hline Gelidiopsis gracilis (Kützin) Vickers & - & - & 6,66 & 0 & - & - & - & - \\
\hline Gelidium pusillum (Stackh.) Le Jolis & 18 & 6 & 15 & 0 & - & - & 25,5 & 0 \\
\hline $\begin{array}{l}\text { Gracilaria domingensis (Kütz.) Sond. ex } \\
\text { Dickie }\end{array}$ & 8 & 3 & - & - & 1 & 10 & - & - \\
\hline Gracilaria sp. & - & - & 18 & 0 & - & - & 22,5 & 0 \\
\hline Griffithsia sp. & - & - & - & - & + & 0 & - & - \\
\hline Herposiphonia tenella (C. Agardh) Hambron & 0,01 & - & - & - & 0,02 & 3 & 6,07 & 2 \\
\hline Hypnea cenomyce J. Agardh & - & - & 0,4 & 0 & 12 & 0 & 5,16 & 1 \\
\hline Hypnea cornuta (Kütz.) J. Agardh & 2 & 0 & - & - & 36 & 0 & - & - \\
\hline $\begin{array}{l}\text { Hypnea musciformis (Wulfen in Jacquin) J. V. } \\
\text { Lamour. }\end{array}$ & 6 & 0 & 7,33 & 3 & 6 & 0 & 25,33 & 1 \\
\hline Jania sp. & - & - & 2 & 0 & - & - & - & - \\
\hline Laurencia obtusa (Huds.)J. V. Lamour. & - & - & 5,5 & 0 & - & - & 42 & 6 \\
\hline Laurencia sp. & & & 3,25 & 0 & - & - & - & - \\
\hline $\begin{array}{l}\text { Osmundaria obtusiloba (C. Agardh) R. E. } \\
\text { Norris }\end{array}$ & 0,04 & 1 & + & 0 & - & - & - & - \\
\hline $\begin{array}{l}\text { Plocamium brasiliense (Grev. in J. St. - Hil.) } \\
\text { M. Howe \& W. R. Taylor }\end{array}$ & - & - & 0,04 & 3 & - & - & - & - \\
\hline Pneophylum fragile Kütz. & 5 & 0 & + & 0 & 20 & 0 & 5 & 0 \\
\hline Polysiphonia subtilissima Mont. & - & - & + & 0 & - & - & - & - \\
\hline $\begin{array}{l}\text { Pterocladiella caerulensis (Kütz.) Santel. \& } \\
\text { Hommers. }\end{array}$ & - & - & - & - & - & - & 19 & 3 \\
\hline Sahlingia subintegra (Rosenv.) Kornmann & - & - & + & 0 & - & - & - & - \\
\hline Stylonema alsidii (Zanardini) K. M. Drew & - & - & + & 0 & - & - & - & - \\
\hline Dictyopteris delicatula J. V. Lamour. (epífita) & 2 & 1 & 17,66 & 0 & 2 & 0 & + & 0 \\
\hline Feldmannia irregularis (Kütz.) Hamel & - & - & 0,25 & 5 & - & - & - & - \\
\hline $\begin{array}{l}\text { Hecatonema floridanum (W. R. Taylor) W. R. } \\
\text { Taylor }\end{array}$ & - & - & + & 0 & - & - & - & - \\
\hline Hecatonema terminalis (Kütz.) Sauv. & - & - & 0,005 & 5 & - & - & - & - \\
\hline Padina gymnospora (Kütz.) Sond. & 0,01 & 10 & & & - & - & - & - \\
\hline Bryopsis pennata J. V. Lamour & 33,50 & 6 & 1 & 0 & 56 & 2 & 0,93 & 0 \\
\hline Bryopsis sp. & - & - & 6,5 & 0 & - & - & - & - \\
\hline Caulerpa sp. & - & - & 16 & 0 & - & - & - & - \\
\hline Cladophora sp. & 0,01 & 0 & & & - & - & - & - \\
\hline $\begin{array}{l}\text { Cladophoropsis membranacea (C. Agardh) } \\
\text { Børgesen (epífita) }\end{array}$ & 0,01 & 0 & 0,155 & 0 & 0,04 & 0 & - & - \\
\hline Halimeda sp. & + & 0 & - & - & - & - & - & - \\
\hline Rhizoclonium riparium (Roth) Kütz. ex Harv. & - & - & + & 0 & - & - & - & - \\
\hline Ulva fasciata Delile & 1 & 10 & - & - & 2 & 10 & - & - \\
\hline Ulva lactuca $\mathrm{L}$. & - & - & 8,1 & 0 & - & - & 3,75 & 0 \\
\hline Ulva rigida C. Agardh & 0,05 & 0 & 0,8 & 0 & 0,02 & 10 & - & - \\
\hline Ulva sp. & - & - & 0,12 & 0 & - & - & - & - \\
\hline
\end{tabular}




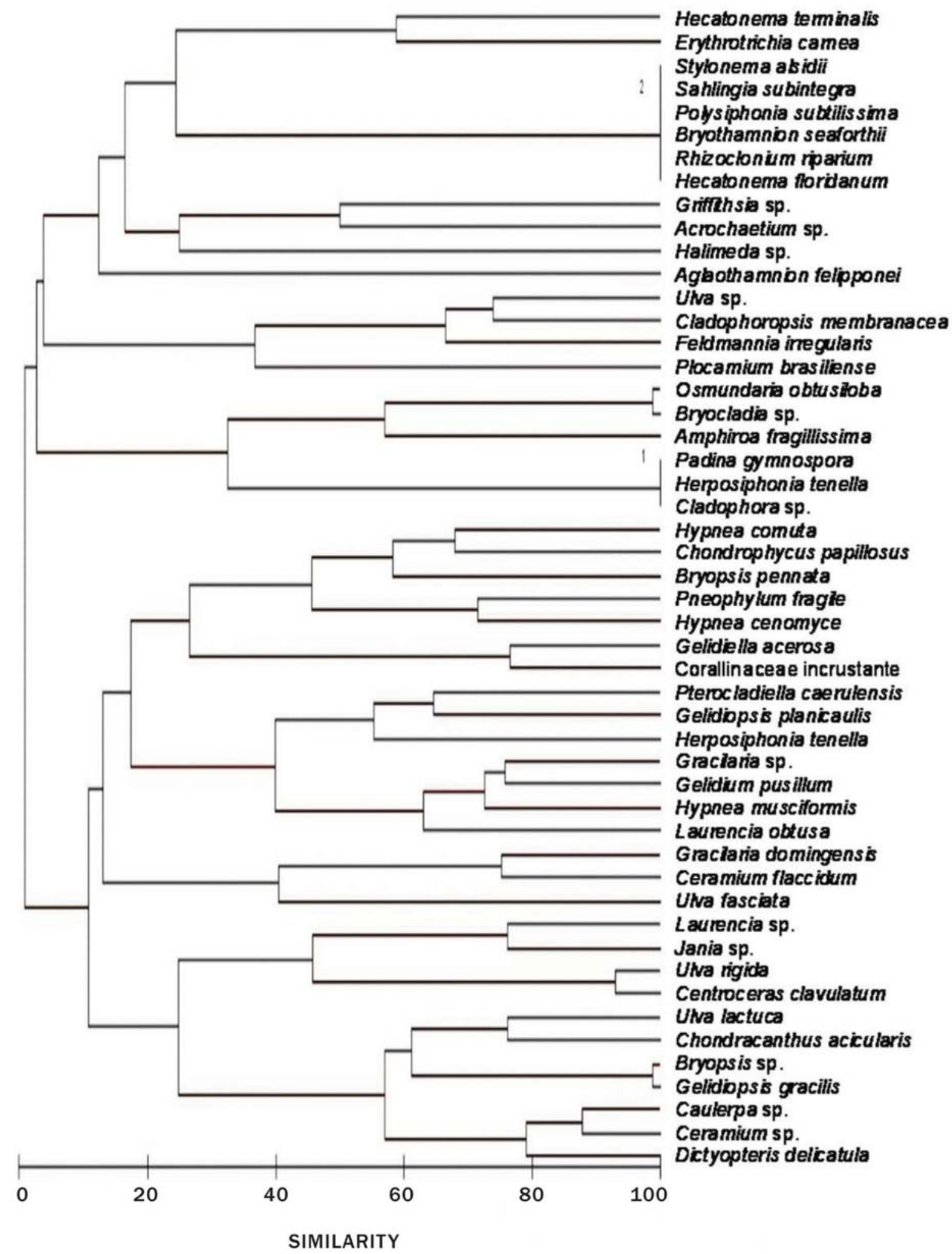

Figura 1. Dendrograma de similaridade sazonal entre as espécies encontradas nos Pontos 1 e 2 do platô recifal da praia de Boa Viagem, Município de Recife - PE.

Figure 1. Cluster analysis of the seasonal similarity among species found in locations 1 and 2 of the plateau of Boa Viagem beach in Recife, Pernambuco state, Brazil. 
Tabela II. Total de espécies (T), Recobrimento Total (Rt), Diversidade específica (H'), Eqüitabilidade (J), e Densidade reprodutiva (dG), calculados para os Pontos 1 e 2, no platô recifal da praia de Boa Viagem, Recife (PE) durante o período chuvoso (abril/2002) e seco (novembro/2003).

Table II. Total number of species (T), total coverage area (Rt), specific diversity $(H)$, equitability $(J)$, and reproductive density $(d G)$ of locations 1 and 2 of the reef plateau of Boa Viagem beach in Recife, Pernambuco state, Brazil, during the rainy period (April 2002) and dry period (November 2003).

\begin{tabular}{|c|c|c|c|c|}
\hline \multirow{3}{*}{ Parâmetros } & \multicolumn{2}{|c|}{ Ponto 1 (Lado Exposto) } & \multicolumn{2}{|c|}{ Ponto 2 (Lado Abrigado) } \\
\hline & Período & Período & Período & Período \\
\hline & chuvoso & seco & chuvoso & seco \\
\hline T (número de espécies) & 26 & 37 & 21 & 18 \\
\hline $\operatorname{Rt}(\%)$ & 267,11 & 187,55 & 340,58 & 345,35 \\
\hline $\mathrm{H}^{\prime}\left(\right.$ bit.cel $^{-1}$.) & 2,62 & 2,22 & 2,52 & 2,53 \\
\hline $\mathrm{J}^{\prime}$ & 0,56 & 0,75 & 0,29 & 0,69 \\
\hline $\mathrm{dG}$ & 3,27 & 1,37 & 0,76 & 0,27 \\
\hline
\end{tabular}

o inverno, no grupo de espécies dominantes constituído por Gracilaria spp. e Caulerpa spp. Segundo esses autores esse fato foi causado pela diminuição da salinidade e aumento dos níveis de fosfato na água.

As diferenças observadas podem ser atribuídas à atuação de um conjunto de fatores abióticos como grau de inclinação do substrato, exposição ao sol e o batimento de ondas (Mansilla \& Pereira 2001). Em relação ao Ponto 1 (Lado Exposto) tais fatores podem ter influenciado num menor recobrimento total, sobretudo o batimento das ondas, que dificulta a fixação de propágulos neste local; provavelmente há um maior investimento em reprodução (maior densidade reprodutiva) que em crescimento vegetativo. O contrário pode ser observado no Ponto 2 (Lado Abrigado).

Com relação ao índice de diversidade específica, tanto o Ponto 1 como o Ponto 2 no período seco e no chuvoso apresentaram uma diversidade média (Yoneshigue \& Valentin 1988), visto que os valores obtidos encontram-se entre 2,0 e 3,0. Com relação à eqüitabilidade os valores encontrados, acima de 0,5 indicam que os indivíduos encontram-se bem distribuídos entre as espécies, exceto no Ponto 2 (período chuvoso) que apresentou um valor de 0,29. Mansilla \& Pereira (2001), encontraram valores de diversidade semelhantes durante o período chuvoso, porém os autores destacam o aumento desse índice no período seco. Com relação aos valores de eqüitabilidade os referidos autores encontraram valores mais elevados, acima de 0,61 .

A análise do dendrograma (Figura 1), obtido com base nos valores de recobrimento, mostra a formação de dois pequenos grupos com $100 \%$ de similaridade. O grupo 1 é formado pelas espécies Cladophora sp., Herposiphonia tenella, Padina gymnospora e o grupo 2 formado por Hecatonema floridanum, Rhizoclonium riparium, Bryothamnion seaforthii, Polysiphonia subti- lissima, Sahlingia subintegra e Stylonema alsidii. A formação desses grupos pode ser resultado do baixo recobrimento e da presença exclusiva em uma das áreas (já que o Grupo 1 é formado por espécies que ocorrem exclusivamente no Ponto 1 e o Grupo 2 incluiu todas as espécies que tiveram recobrimentos não mensurados, e ocorreram apenas no Ponto 1 no período seco).

Apesar das formações recifais da praia de Boa Viagem estarem sujeitas a uma ação antrópica negativa, a flora ficológica apresentou um padrão florístico correspondente ao encontrado em outras áreas mais preservadas da costa pernambucana como Enseada dos Corais (Pereira et al. prelo) e Cupe (Mansilla \& Pereira 2001).

AGRADECIMENTOS: Os autores expressam seus agradecimentos ao Programa de Pós-Graduação em Botânica (PPGB) da Universidade Federal Rural de Pernambuco, pelo apoio financeiro. Ao Conselho Nacional de Desenvolvimento Científico e Tecnológico (CNPq) e a Coordenação de Aperfeiçoamento de Pessoal de Nível Superior (CAPES) pelas bolsas concedidas. Ao Dr. Paulo Antunes Horta, da Universidade Federal de Santa Cantarina, pela utilização do Programa PRIMER.

\section{REFERÊNCIAS BIBLIOGRÁFICAS}

BANDEIRA-PEDROSA, M.E.; PEREIRA, S.M.B \& OLIVEIRA, E.C. 2004. Taxonomy and distribution of the green algal genus Halimeda (Bryopsidales, Chlorophyta) in Brazil. Revista Brasileira de Botânica, 27(2): 363-377.

COSTA JR, O.S.; ATTRILL, M.J.; PEDRINI, A.G. \& DEPAULA, J.C. 2002. Spatial and seasonal Distribution of seaweeds on coral reefs from Southern Bahia, Brazil. Botanica Marina, 45: 346-355.

FERREIRA, M.V; PEREIRA, S.M.B; CARVALHO, F.A.F; TEIXEIRA, G.C; GUEDES, E.A.C; PAES e MELO, L.B; MATTOS, S.M.G.; SILVA, R.L; PEDROSA, M.E.B \& CARVALHO, G.V.S. 1988. Prospecção dos bancos de algas marinhas dos Estados da Paraíba, de Pernambuco, e de Alagoas (Profundidade de 0 a 10m). Gayana Botanica, 45(1-4): 413-422.

GUERRA, N.C. \& MANSO, V.A. 2004. Beachrocks (Recifes de Arenito). Pp 109-130. In: E. Eskinazi-Leça, S. Neumann-Leitão \& M. F. Costa, (orgs.), Oceanografia: Um cenário tropical. Bagaço, Recife.761p. 
HORTA, P.A.; AMANCIO, E.; COIMBRA, C.S. \& OLIVEIRA, E.C. 2001. Considerações sobre a distribuição e origem da flora de macroalgas marinhas brasileiras. Hoehnea, 28(3): 243-265.

KEMPF, M. 1967/69. A plataforma continental de Pernambuco (Brasil): Nota preliminar sobre a natureza do fundo. TrabaIhos Oceanográficos da Universidade Federal de Pernambuco, 9/11: 111-124.

MANSILLA, A. \& PEREIRA, S.M.B. 2001. Comunidades y diversidad de macroalgas em pozas intermareales de arrecifes. Pp 315-330. In: K. Alneval \& T. Antezana, (eds.), Sustentabilidad de la Biodiversidad, un problema actual. Bases científico-técnicas teorizaciones y proyecciones. Universidad de Concepción, Chile. 896p.

MUÑOZ, A.O.M. \& PEREIRA, S.M.B. 1998. Estrutura de comunidades de macraolgas em formações recifais do Estado de Pernambuco, Brasil. v. II. Pp 287-297. In: E. J. Paula; M. Cordeiro-Marino; D. P. Santos.; E. M. Plastino, M.T. Fujii \& N.S. Yokoya, (eds.), Anais do IV Congresso Latino-Americano, II Reunião Ibero- Americana, VII Reunião Brasileira de Ficologia. Conservação da Biodiversidae e Novas Tecnologias: Promessas e Perigos. Sociedade Brasileira de Ficologia. São Paulo. 495p.

OLIVEIRA-CARVALHO, M.F.; PEREIRA, S.M.B. \& ZICKEL, C.S. 2003. Florística e distribuição espaço- temporal das clorofíceas bentônicas em trechos recifais do litoral norte do estado de Pernambuco. Hoehnea, 30(3): 201-212.

OLIVEIRA FILHO, E.C. 1977. Algas marinhas bentônicas do Brasil. Tese de Livre Docência, Universidade de São Paulo, São Paulo, Brasil. 407p.

PEREIRA, S.M.B. 2002. Desenvolvimento e situação atual do conhecimento das macroalgas marinhas das regiões Norte e Nordeste. Pp 117-121. In: E.L. Araújo, A.N. Moura, E.V.S.B. Sampaio, L.M.S. Gestinari \& J.M.T. Carneiro, (eds.), Biodiversidade, conservação e uso sustentável da flora do Brasil. Editora Universitária, Recife. 262p.

PEREIRA, S.M.B; OLIVEIRA-CARVALHO, M.F.; ANGEIRAS, J.A.P.; BANDEIRA-PEDROSA, M.E.; OLIVEIRA, N.M.B.; TORRES, J.; GESTINARI, L.M.S.; COCENTINO, A.L.M.; SANTOS, M.D.; NASCIMENTO, P.R.F. \& CAVALCANTI, D.R. 2002. Algas Marinhas Bentônicas do Estado de Pernambuco. v. 1, Pp 97-124. In: M. Tabarelli \& J.M.C. Silva, (orgs.), Diagnóstico da Biodiversidade de Pernambuco. Secretaria de Ciência, Tecnologia e Meio Ambiente, Recife. 356p.

PEREIRA, S.M.B; OLIVEIRA-CARVALHO, M.F; BURGOS, D.C.\& ARAÚJO, E.L. (no prelo). Caracterização estrutural das macroalgas de ambiente recifal da praia de Enseada dos Corais- Pernambuco- Brasil. In: Anais do XI Congresso Brasileiro de Ficologia. Série Livros do Museu Nacional, v. 30.

SILVA, R.L.; PEREIRA, S.M.B.; OLIVEIRA F.E.C. \& ESTON, V.R. 1987. Structure of a bed of Gracilaria spp. (Rhodophyta) in northeastern Brazil. Botanica Marina, 30: 517-523.

YONESHIGUE, Y. \& VALENTIN, J.V. 1988. Comunidades algais fotófilas de infralitoral de Cabo Frio, Rio de Janeiro, Brasil. Gayana Botânica, 45: 61-75.
VILLAÇA, R.; PEDRINI, A.G.; PEREIRA, S.M.B. \& FIGUEREDO, M.A.O. 2006. Flora marinha bentônica das ilhas oceânicas brasileiras. Pp105-146. In: R.J.V. Alves \& J.W.A. Castro, (orgs.), Ilhas Oceânicas Brasileiras: da pesquisa ao manejo. Ministério do Meio Ambiente. Brasília. 298p.

Submetido em 15/01/2008. Aceito em 21/03/2008. 